\title{
The charged exciton in an InGaN quantum dot on a GaN pyramid
}

\author{
Chih-Wei Hsu, Evgenii Moskalenko, Martin Eriksson, Anders Lundskog, Fredrik K. \\ Karlsson, Urban Forsberg, Erik Janzén and Per-Olof Holtz
}

\section{Linköping University Post Print}

N.B.: When citing this work, cite the original article.

Original Publication:

Chih-Wei Hsu, Evgenii Moskalenko, Martin Eriksson, Anders Lundskog, Fredrik K. Karlsson, Urban Forsberg, Erik Janzén and Per-Olof Holtz, The charged exciton in an InGaN quantum dot on a GaN pyramid, 2013, Applied Physics Letters, (103), 1.

http://dx.doi.org/10.1063/1.4812984

Copyright: American Institute of Physics (AIP) http://www.aip.org/

Postprint available at: Linköping University Electronic Press http://urn.kb.se/resolve?urn=urn:nbn:se:liu:diva-95961 


\title{
The charged exciton in an InGaN quantum dot on a GaN pyramid
}

\author{
Chih-Wei Hsu, ${ }^{1, a)}$ Evgenii S. Moskalenko, ${ }^{1,2}$ Martin O. Eriksson, ${ }^{1}$ Anders Lundskog, ${ }^{1}$ \\ K. Fredrik Karlsson, ${ }^{1}$ Urban Forsberg, ${ }^{1}$ Erik Janzén, ${ }^{1}$ and Per Olof Holtz ${ }^{1}$ \\ ${ }^{1}$ Department of Physics, Chemistry and Biology (IFM), Linköping University, SE-581 83 Linköping, Sweden \\ ${ }^{2}$ A. F. Ioffe Physical-Technical Institute, RAS, 194021, Polytechnicheskaya 26, St. Petersburg, Russia
}

(Received 22 April 2013; accepted 16 June 2013; published online 2 July 2013)

\begin{abstract}
The emission of a charged exciton in an InGaN quantum dot (QD) on top of a GaN pyramid is identified experimentally. The intensity of the charged exciton exhibits the expected competition with that of the single exciton, as observed in temperature-dependent micro-photoluminescence measurements, performed with different excitation energies. The non-zero charge state of this complex is further supported by time resolved micro-photoluminescence measurements, which excludes neutral alternatives of biexciton. The potential fluctuations in the vicinity of the QD that localizes the charge carriers are proposed to be responsible for the unequal supply of electrons and holes into the QD. () 2013 AIP Publishing LLC. [http://dx.doi.org/10.1063/1.4812984]
\end{abstract}

Besides the exploration of new physical properties in quantum dots (QDs), the proposed quantum information applications (QIA), which make use of the quantum states of particles to transmit and encode information, also motivate the research on QDs. QDs are considered as a promising photon source which fulfill the essential demand for QIA: the generation of photons with specific energy polarization- and time-correlations. ${ }^{1}$ A quantum photon source relies on the realization and engineering of specific excitonic states of various charge configurations of the QD. From the perspective of materials, III-nitrides QDs can be advantageous over III-arsenide counterparts due to their very wide spectral tunability of the photon energy and possible deep confining potentials for high temperature operation. ${ }^{2}$ However, the development of high-quality III-nitride-based QDs today is well behind III-arsenide-based QDs. Regarding the fundamental identification of excitonic states, single excitons and biexcitons have been identified in the micro-photoluminescence ( $\mu \mathrm{PL}$ ) spectra of GaN and InGaN QDs, mainly based on their expected linear and quadratic intensity dependencies on the excitation power. ${ }^{3-5}$ In some reports, this identification was further strengthened by more robust signatures of the exciton/ biexciton pair, such as congruent fine structure splittings ${ }^{6}$ or photon bunching characteristics. ${ }^{2}$ The trion states have so far not been identified for the nitride-based QDs.

Direct growth of QDs on pre-fabricated templates of micro-/nano-structures has been demonstrated as an effective approach for controlling the position of the QDs for various systems. ${ }^{4,7-9}$ By employing such spatially isolated QDs, the typical large inhomogeneous broadening, as well as dot-todot interactions, can be avoided. ${ }^{10}$ This facilitates the spectral identification, ${ }^{11}$ and a detailed polarized fine structure has been revealed in III-As QDs. ${ }^{12}$ The InGaN QDs presented in this letter, exhibiting sub-meV emission lines in $\mu \mathrm{PL}$, were formed individually on top of $\mathrm{GaN}$ pyramids. One emission line of a single InGaN QD in this work is consistently attributed to a trion, despite its quadratic excitation power dependence revealed at low temperatures $(\sim 4 \mathrm{~K})$. It is

\footnotetext{
${ }^{\text {a) }}$ Author to whom correspondence should be addressed. Electronic mail: cwhsu@ifm.liu.se
}

demonstrated that the power dependence solely is not sufficient for reliable identification and that the temperature dependence is an important complement to enable a conclusion about the origin of the excitonic emission lines.

A low-temperature $\mu \mathrm{PL}$ system with two continuouswave (cw) lasers, providing two excitation energies at $h v_{e x}=4.66 \mathrm{eV}$ and $h v_{e x}=3.49 \mathrm{eV}$, respectively, was employed to perform the power $\left(P_{e x}\right)$ - and temperature $(T)$-dependent $\mu \mathrm{PL}$ measurements. Since the $\mathrm{InGaN}$ layer was sandwiched between $\mathrm{GaN}$ layers, the band gap of $\mathrm{GaN}(3.51 \mathrm{eV})$ represents the maximum energy of the barrier material confining the InGaN QDs, i.e., $h v_{e x}=4.66 \mathrm{eV}$ and $h v_{e x}=3.49 \mathrm{eV}$ correspond to above and near-resonant barrier excitation, respectively. A Ti:sapphire laser coupled in series with a second harmonic generator was applied to perform the $\mu \mathrm{PL}$ excitation ( $\mu \mathrm{PLE}$ ) spectroscopy in the energy range of $3.13-3.51 \mathrm{eV}$. Time resolved $\mu \mathrm{PL}$ (TR $\mu \mathrm{PL})$ experiments were performed with excitation pulses (200 fs pulse width and $75 \mathrm{MHz}$ repetition rate) at $h v_{e x}=4.66 \mathrm{eV}$ generated by a frequency-tripled Ti-sapphire laser. A streak camera was used to acquire the transient PL signal with a time resolution of $10 \mathrm{ps}$.

Two individual QDs (QD1 and QD2), located at two different pyramids with different emission features, are presented for comparison. For QD1, only one emission line, denoted as $\mathrm{X}_{0}$, is observed, and no other emission line appears when varying $P_{e x}$ and $T$ (Figs. 1(a) and 1(b), respectively). $P_{e x}$-dependent $\mu \mathrm{PL}$ performed on QD1 at $4 \mathrm{~K}$ reveals that the intensity of $\mathrm{X}_{0}$ is directly proportional to $P_{e x}$, as characteristic for the single exciton. It should be noted that most QDs we studied exhibit only the single exciton emission. The presence of merely a single exciton can be ascribed to a shallow confinement potential in which solely the single neutral exciton state is bound. ${ }^{9}$ Other excitonic complexes in III-nitride QDs, like the charged exciton and biexciton, are expected to appear at higher energies than the single exciton due to the strong repulsive Coulomb interactions, thus being unbound for the case of shallow barriers. ${ }^{13}$

In QD2, only one emission line, denoted as $\mathrm{X}_{\mathrm{A}}$, is recorded at low $P_{e x}$ and/or $T$. However, an additional emission line, denoted as $\mathrm{X}_{\mathrm{B}}$, appears with increasing $P_{e x}$ and/or $T$ (Fig. 1). According to our polarization-dependent 


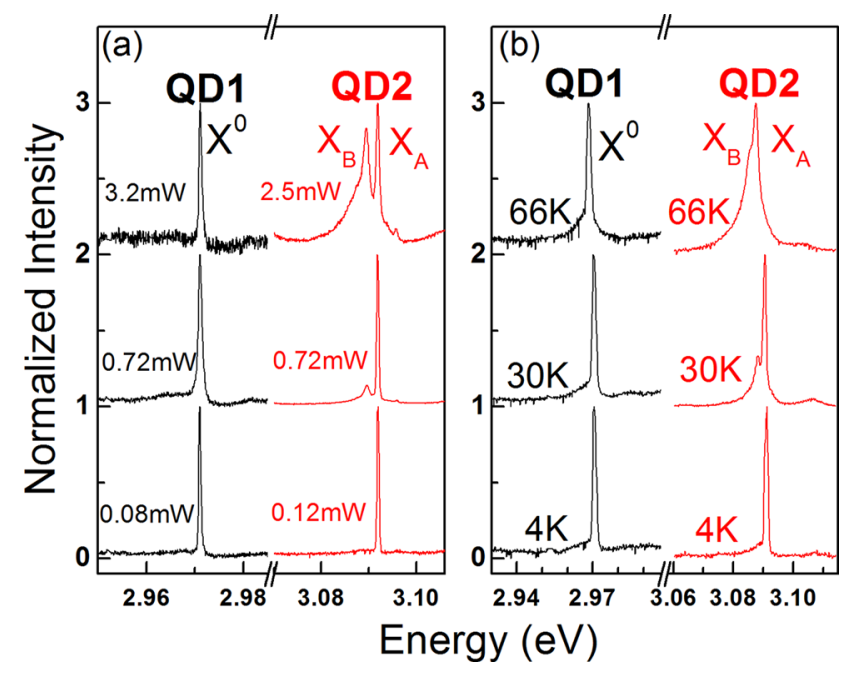

FIG. 1. $\mu \mathrm{PL}$ spectra of QD1 and QD2 employing the excitation energy $h v_{e x}=4.66 \mathrm{eV}$ at (a) varying excitation power at constant temperature, $4 \mathrm{~K}$, (b) varying temperature at constant excitation power of $0.7 \mathrm{~mW}$. The spectra of QD1 are normalized to the intensity of $\mathrm{X}_{0}$ while the spectra of QD2 are normalized to the intensity of $\mathrm{X}_{\mathrm{A}}$. They are all shifted along the $\mathrm{y}$-axis for clarity.

measurements, $\mathrm{X}_{\mathrm{A}}$ and $\mathrm{X}_{\mathrm{B}}$, are linearly polarized in the same direction and with a similar degree of polarization, ${ }^{14}$ which can be interpreted as two different exciton complexes originating from the same QD involving the same hole state. ${ }^{15}$ The intensity of $\mathrm{X}_{\mathrm{A}}\left(I_{X A}\right)$ is linearly dependent on the $P_{e x}$ at $4 \mathrm{~K}$ (Fig. 2(a)), by which $\mathrm{X}_{\mathrm{A}}$ can be ascribed to the single exciton. The intensity of $\mathrm{X}_{\mathrm{B}}\left(I_{X B}\right)$ exhibits a superlinear (quadratic) dependence on $P_{e x}$ (Fig. 2(a)), suggesting that $\mathrm{X}_{\mathrm{B}}$ is associated with an exciton complex consisting of more than one electron and one hole. The superlinear $P_{e x}$ dependence is another indication that $\mathrm{X}_{\mathrm{A}}$ and $\mathrm{X}_{\mathrm{B}}$ originate from the same QD; If $\mathrm{X}_{\mathrm{B}}$ would be a single exciton of another neighboring QD, a linear dependence on $P_{e x}$ would be expected.

A quadratic $P_{e x}$ dependence is commonly understood as the signature of the biexciton. However, we will argue that $\mathrm{X}_{\mathrm{B}}$ should not be identified as the biexciton despite the

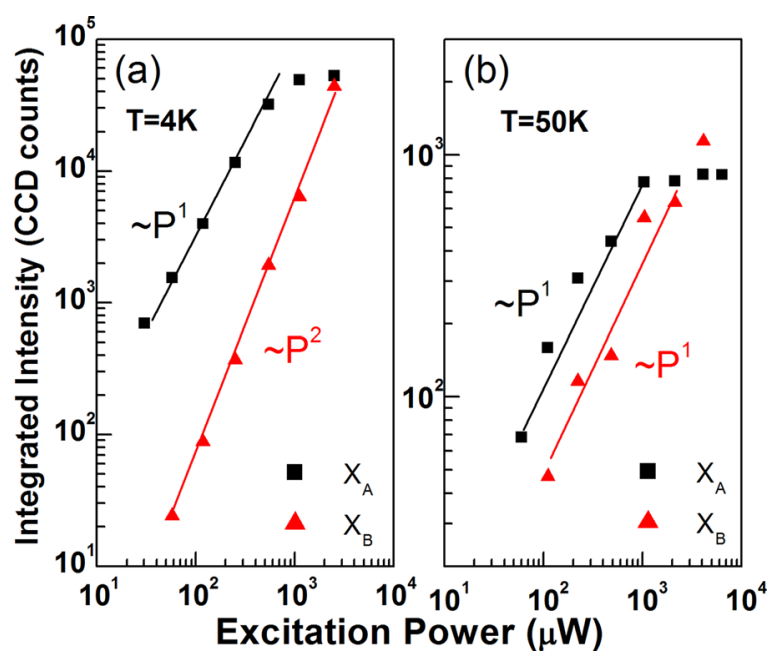

FIG. 2. The integrated emission intensities of $X_{A}$ and $X_{B}$ as a function of applied excitation powers plotted in logarithmic scales performed at (a) $4 \mathrm{~K}$, (b) $50 \mathrm{~K}$. The excitation energy was $h v_{e x}=4.66 \mathrm{eV}$. The experimental results are shown in scatters. The solid lines with indicated slopes are shown for visual clarity. quadratic power dependence of $I_{X B}$ observed at $4 \mathrm{~K}$. First, the quadratic $P_{e x}$-dependence of $\mathrm{X}_{\mathrm{B}}$ at $4 \mathrm{~K}$ turns into an almost linear dependence at $50 \mathrm{~K}$ (Fig. 2(b)) whereas the linear $P_{e x}$-dependence of $\mathrm{X}_{\mathrm{A}}$ at $4 \mathrm{~K}$ remains linear at $50 \mathrm{~K}$ (Figs. 2(a) and 2(b)). Thus, the experimental observation of linear $P_{e x}$-dependence for both $I_{X A}$ and $I_{X B}$ at $50 \mathrm{~K}$ contradicts the arguments of $I_{X B}$ being the biexciton. Moreover, for a biexciton-exciton cascade recombination under pulsed excitation, the recombination of a biexciton will leave an exciton as the intermediate state, with subsequent exciton emission. If the probability of populating the QD with two $e$ - $h$ pairs (biexciton) is significantly high, a delayed transient profile for the exciton with respect to that for the biexciton is expected. According to reported models of the biexcitonexciton system, the decay curve of the single exciton can be quantitatively described by rate equations. ${ }^{16,17}$ In our case, the measured time-dependent $I_{X A}$ does not match well with the modeled decay curve, implying that the assumption of $\mathrm{X}_{\mathrm{B}}$ being a biexciton is false. ${ }^{14}$ Finally, due to the sequential recombination of the biexciton-exciton system, an additive rather than a competitive emission intensity dependence is expected. A pronounced competition in terms of recombination probability between $\mathrm{X}_{\mathrm{A}}$ and $\mathrm{X}_{\mathrm{B}}$ is observed in our $T$-dependent measurements, which accordingly is inconsistent with the interpretation of $\mathrm{X}_{\mathrm{B}}$ and $\mathrm{X}_{\mathrm{A}}$ as the signatures of a biexciton-exciton system.

Fig. 3(a) shows the full spectrum of the GaN pyramid hosting QD2; exhibiting features related to the GaN barriers, QD2 and the InGaN layers on the facets of the GaN pyramid. The low density of states of the QDs makes it highly unlikely for absorption of photons directly into the QD volume. ${ }^{18}$ Consequently, the emission of the QD is mainly the result of an excitation, in which the carriers are subsequently trapped into the QD from its surrounding barriers. From the $\mu$ PLE spectrum in Fig. 3(a), it is demonstrated that $h v_{e x}$ above $\sim 3.1 \mathrm{eV}$ can contribute to $I_{Q D 2}$. This threshold energy observed is likely originating from potential fluctuations present in the vicinity of QD2. The probability for carriers to become captured by QD2 could be reduced because the carriers may be localized in these potential fluctuations. Such effects could be qualitatively investigated by selective generation of excited carriers, performed by means of different excitation energies at different temperatures. With $h v_{e x}=4.66 \mathrm{eV}$, a progressive enhancement of the integrated emission intensity of QD2 $\left(I_{Q D 2}=I_{X A}+I_{X B}\right)$ with increasing $T$ is observed (Fig. $3(\mathrm{~b})$ ), indicating that QD2 receives additional carriers ( $e$ and $h$ ) from its vicinity via thermal excitation of localized carriers. ${ }^{19}$ It should be noted that $P_{e x}$ of the laser was tuned to acquire an intensity of $\mathrm{X}_{\mathrm{B}}$ "just above the detectable limit" with an initial intensity ratio of $I_{X B} / I_{X A} \sim 0.07$ at $4 \mathrm{~K}$ (Fig. 3(c)), ensuring that $I_{X A}$ and/or $I_{X B}$ are not saturated by $P_{e x}$. The increasing ratio of $I_{X B} / I_{X A}$ with increasing $T$ (Fig. 3(c)) agrees with the interpretation of $X_{\mathrm{B}}$ as being an excitonic complex because the probability for QD2 to acquire more than a sole $e-h$ pair should increase with increasing $T$. On the other hand, the $I_{Q D 2}$ remains essentially constant with increasing $T$ under $h v_{e x}=3.49 \mathrm{eV}$ (Fig. 3(b)), indicating that QD2 does not receive extra thermally activated $e-h$ pairs for enhancing $I_{Q D 2}$. The combination of an observed constant $I_{Q D 2}$ and an increasing ratio of $I_{X B} / I_{X A}$ with increasing $T$ reveals that $I_{X A}$ is suppressed and $I_{X B}$ is 


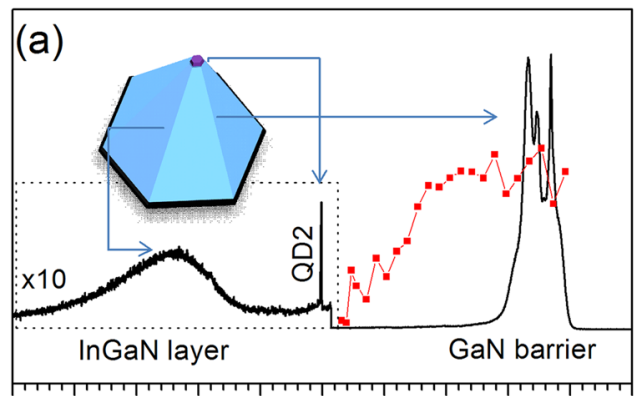

2.62 .72 .82 .93 .03 .13 .23 .33 .43 .53 .6 Energy (eV)
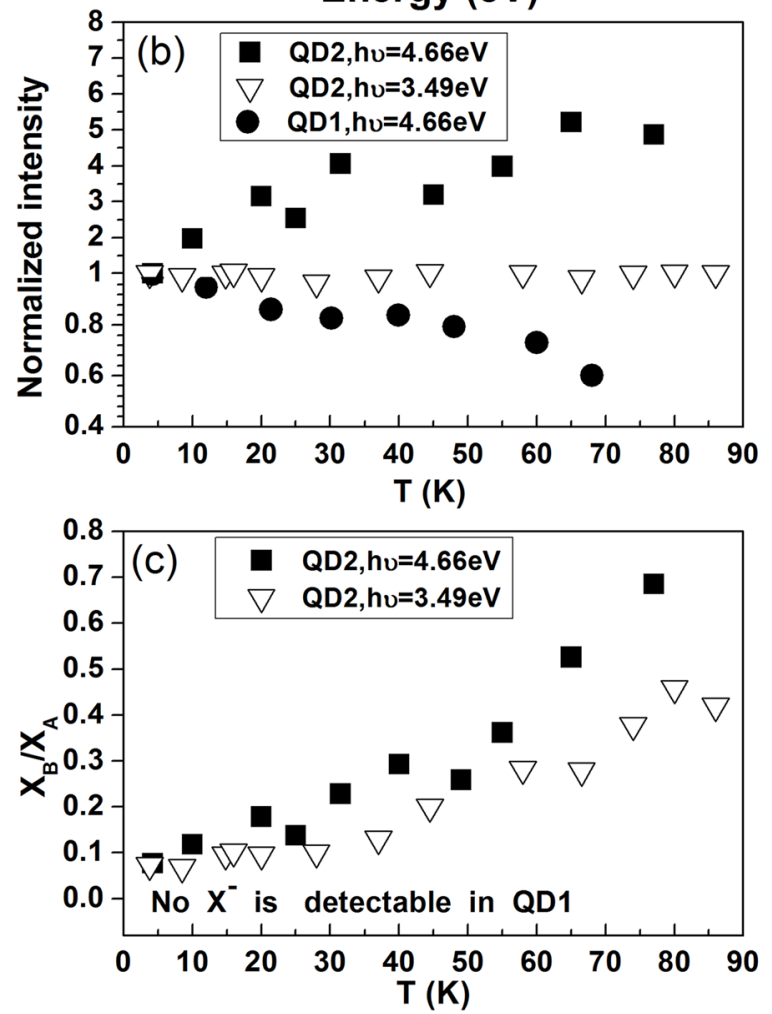

FIG. 3. (a) $\mu \mathrm{PL}$ spectra of QD2 together with its hosting pyramid and InGaN layer formed on the facets as indicated in the figure. The emission intensity within the dash-line frame is multiplied by a factor of 10 for clarity. The $\mu$ PLE spectrum of the integrated emission intensity of QD2 is shown in red color. (b) Plots of the integrated emission intensity at different temperatures with two different excitation energies $\left(h v_{e x}=4.66 \mathrm{eV}\right.$ and $h v_{e x}=3.49 \mathrm{eV}$ ). The results are normalized to the intensity recorded at $4 \mathrm{~K}$. Note that the $y$-scale changes for visual clarity when the value is smaller than 1. (c) Plots of the ratio of $X_{B} / X_{A}$ at different temperatures with excitation energies both at $h v_{e x}=4.66 \mathrm{eV}$ and $h v_{e x}=3.49 \mathrm{eV}$.

enhanced with increasing $T$. Such an observation is inconsistent with the general understanding of a biexciton-exciton recombination scheme in which the single exciton would increase together with the biexciton in an additive way for the case of low $P_{e x}$, well below the single exciton saturation as used in these experiments. Instead, the competing behavior observed for $I_{X A}$ and $I_{X B}$ strongly implies that both $\mathrm{X}_{\mathrm{A}}$ and $\mathrm{X}_{\mathrm{B}}$ originate from excitons involving just one e-h pair, but $\mathrm{X}_{\mathrm{B}}$ should involve an extra charge carrier.

Charged excitons are formed due to unequal capture/ supply rates of electrons and holes from the potential barrier into the QD. The difference in carrier relaxation times and diffusivities between electrons and holes, dopants/impurities and potential fluctuations in the vicinity of the QD can be

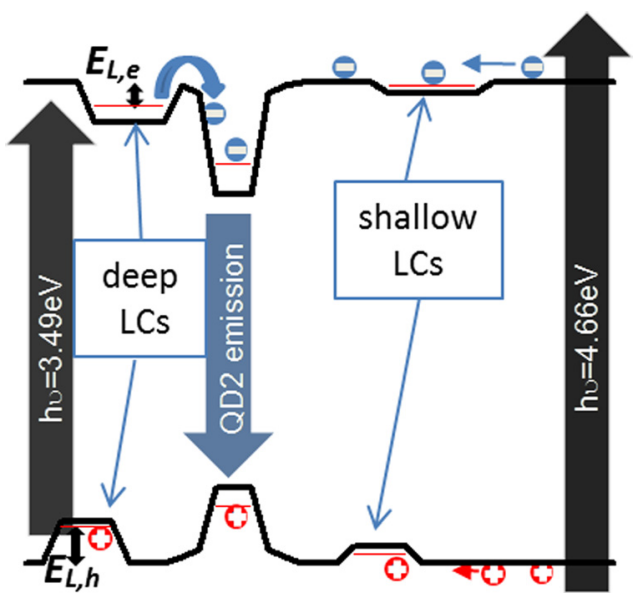

FIG. 4. A schematic figure illustrating the source of additional charge carrier involved in the recombination of $\mathrm{X}_{\mathrm{B}}$ by considering the potential fluctuations in the vicinity of QD2. Upon excitation with $h v_{e x}=3.49 \mathrm{eV}$, only QD2 and deeper potential fluctuations are populated. Only electrons localized in the populated potential fluctuation can overcome the ionization energy $\left(E_{L}\right)$ and then be recaptured by QD2 as illustrated in the figure.

utilized to manipulate the exciton charge states by means of varying the excitation energies, excitation power, and temperature. ${ }^{11,20}$ Carriers localized in such potential fluctuations must acquire sufficient energy to overcome the energy barrier to become captured into the QD. The escape probability of carriers from the potential fluctuations is proportional to $\exp \left(-E_{L} / k_{B} T\right)$, where $k_{B}$ is the Boltzmann constant and $E_{L}$ is the ionization energy of the confined carriers. Given that the effective e mass is about $1 / 5$ with respect to the effective $h$ mass in GaN (Ref. 21) and assuming the potential depths are comparable for the e and the $\mathrm{h}$, the e should have significantly larger probability to escape and subsequently be captured by QD2, implying a negatively charged exciton for $\mathrm{X}_{\mathrm{B}}$. A proposed recombination scheme involving the potential fluctuations (Fig. 4) can be explained in the following way: By $h v_{e x}=4.66 \mathrm{eV}$, the excited carriers gain sufficiently high energy to populate QD2, all potential fluctuations and the GaN barrier. The continuously enhanced $I_{Q D 2}$ with increasing $T$ (Fig. 3(b)) indicates a considerable enhancement of the escape probabilities for both $\mathrm{e}$ and $\mathrm{h}$ and a thermally facilitated diffusivity of carriers in the barriers at higher $T$. On the other hand, for $h v_{e x}=3.49 \mathrm{eV}$, only QD2 and the deeper potential fluctuations with energy gaps smaller than $h v_{e x}=3.49 \mathrm{eV}$ are populated. The route for QD2 to acquire carriers in addition to the weak direct excitation is the thermally activated process of localized carriers from potential fluctuations. As stated above, $\mathrm{X}_{\mathrm{B}}$ must possess an additional charge compared to $\mathrm{X}_{\mathrm{A}}$ in order to explain the experimental observations, which is believed to be an e.

Theoretical models of strained III-nitride QDs with built in electric fields generally predict a certain order of the emission energies of the exciton complexes according to $E_{X X}>E_{X^{+}}>E_{X^{-}}$. The reason for this trend is that the repulsive Coulomb interaction between holes is larger than the corresponding repulsion between electrons, which in turn is larger than the attractive electron-hole interaction $\left(\left|\mathrm{J}_{\mathrm{hh}}\right|>\left|\mathrm{J}_{\mathrm{ee}}\right|>\left|\mathrm{J}_{\mathrm{eh}}\right|\right){ }^{13}$ All reported models predict negative binding energies of the complexes $\mathrm{XX}, \mathrm{X}^{+}$, and $\mathrm{X}^{-}$, but Coulomb correlations are known to redshift the emission 
energies with respect to the single exciton $\mathrm{X}$. Thus, a plausible explanation to why the current models do not exhibit any positive binding energies, as sometimes observed experimentally, is that the correlation effects have not been taken into account in a realistic way in the models proposed. Under a successive redshift of $\mathrm{XX}, \mathrm{X}^{+}$, and $\mathrm{X}^{-}$with respect to $X$, the negatively charged exciton $X^{-}$is the first one that turns from a negative binding energy into a positive binding energy. It should also be noted that the computational predictions on the polarization degree for exciton complexes predict a negligible difference in the polarization degree between the single exciton and the negatively charged exciton, whereas a small, but noticeable difference is typically found between the single exciton and the positively charged exciton and the biexciton. ${ }^{14}$ Accordingly, the negatively charged exciton is the most likely interpretation of $X_{B}$.

In conclusion, several optical characterization techniques, including $\mu \mathrm{PL}, \mu \mathrm{PLE}$, and $\mathrm{TR} \mu \mathrm{PL}$, were employed to investigate the origins of two emission lines $\left(\mathrm{X}_{\mathrm{A}}\right.$ and $\left.\mathrm{X}_{\mathrm{B}}\right)$ from single InGaN QDs on a GaN pyramid. The emission lines are believed to be the single exciton and the negativelycharged exciton based on the following remarks: (i) Both $\mathrm{X}_{\mathrm{A}}$ and $X_{B}$ are verified to originate from the same QD. Powerand polarization-dependent measurements suggest that $\mathrm{X}_{\mathrm{A}}$ and $\mathrm{X}_{\mathrm{B}}$ are a single exciton and an exciton complex, respectively. (ii) The option of a biexciton for $\mathrm{X}_{\mathrm{B}}$ is excluded and instead a three-particle configuration is suggested because the recombination of a biexciton is not expected to compete but rather be additive with the recombination of the single exciton. TR $\mu \mathrm{PL}$ results are not consistent with an interpretation of $\mathrm{X}_{\mathrm{A}}$ and $\mathrm{X}_{\mathrm{B}}$ as being biexciton-exciton, as the expected delay of the exciton relative to the biexciton is not observed. (iii) The negatively charged exciton is suggested based on the escape probability of localized carriers from the potential fluctuations in the vicinity of the QD and the comparison between our experimental results and theoretical predictions of emission polarization properties associated with various exciton complexes from a QD.
The authors would like to thank for the financial support from the NANO-N consortium funded by the Swedish Foundation for Strategic Research (SSF).

${ }^{1}$ A. J. Shields, Nat. Photonics 1, 215 (2007).

${ }^{2}$ S. Kako, C. Santori, K. Hoshino, S. Götzinger, Y. Yamamoto, and Y. Arakawa, Nat. Mater. 5, 887 (2006).

${ }^{3}$ S. Kako, K. Hoshino, S. Iwamoto, S. Ishida, and Y. Arakawa, Appl. Phys. Lett. 85, 64 (2004).

${ }^{4}$ J. Renard, R. Songmuang, C. Bougerol, D. Daudin, and B. Gayral, Nano Lett. 8, 2092 (2008).

${ }^{5}$ R. Bardoux, A. Kaneta, M. Funato, Y. Kawakami, A. Kikuchi, and K. Kishino, Phys. Rev. B 79, 155307 (2009).

${ }^{6}$ S. Amloy, Y. T. Chen, K. F. Karlsson, K. H. Chen, H. C. Hsu, C. L. Hsiao, L. C. Chen, and P. O. Holtz, Phys. Rev. B 83, 201307 (2011).

${ }^{7}$ Y. Sugiyama, Y. Sakuma, S. Muto, and N. Yokoyama, Appl. Phys. Lett. 67, 256 (1995).

${ }^{8}$ A. Hartmann, L. Loubies, F. Reinhardt, and E. Kapon, Appl. Phys. Lett. 71, 1314 (1997).

${ }^{9}$ C. W. Hsu, A. Lundskog, K. F. Karlsson, U. Forsberg, E. Janzén, and P. O. Holtz, Nano Lett. 11, 2415 (2011).

${ }^{10}$ E. S. Moskalenko, K. F. Karlsson, P. O. Holtz, B. Monemar, W. V. Schoenfeld, J. M. Garcia, and P. M. Petroff, Phys. Rev. B 64, 085302 (2001).

${ }^{11}$ A. Hartmann, Y. Ducommun, E. Kapon, U. Hohenester, and E. Molinari, Phys. Rev. Lett. 84, 5648 (2000).

${ }^{12}$ K. F. Karlsson, M. A. Dupertuis, D. Y. Oberli, E. Pelucchi, A. Rudra, P. O. Holtz, and E. Kapon, Phys. Rev. B 81, 161307 (2010).

${ }^{13}$ D. P. Williams, A. D. Andreev, and E. P. O'Reilly, Superlattices Microstruc. 36, 791 (2004).

${ }^{14}$ See supplementary material at http://dx.doi.org/10.1063/1.4812984 for further discussions.

${ }^{15}$ S. Amloy, K. F. Karlsson, T. G. Andersson, and P. O. Holtz, Appl. Phys. Lett. 100, 021901 (2012).

${ }^{16}$ J. H. Rice, J. W. Robinson, J. H. Na, K. H. Lee, R. A. Taylor, D. P. Williams, E. P. O'Reilly, A. D. Andreev, Y. Arakawa, and S. Yasin, Nanotechnology 16, 1477 (2005).

${ }^{17}$ G. Bacher, R. Weigand, J. Seufert, V. D. Kulakovskii, N. A. Gippius, A. Forchel, K. Leonardi, and D. Hommel, Phys. Rev. Lett. 83, 4417 (1999).

${ }^{18}$ E. S. Moskalenko, K. F. Karlsson, V. Donchev, P. O. Holtz, W. V. Schoenfeld, and P. M. Petroff, Appl. Phys. Lett. 84, 4896 (2004).

${ }^{19}$ E. S. Moskalenko, K. F. Karlsson, V. T. Donchev, P. O. Holtz, B. Monemar, W. V. Schoenfeld, and P. M. Petroff, Nano Lett. 5, 2117 (2005).

${ }^{20}$ E. S. Moskalenko, K. F. Karlsson, P. O. Holtz, B. Monemar, W. V. Schoenfeld, J. M. Garcia, and P. M. Petroff, J. Appl. Phys. 92, 6787 (2002).

${ }^{21}$ I. Vurgaftman and J. R. Meyer, J. Appl. Phys. 94, 3675 (2003). 